\title{
Determination of forest road surface roughness by Kinect depth imaging
}

\author{
F. Marinello, A.R. Proto, G. Zimbalatti, A. Pezzuolo, R. Cavalli, S. Grigolato
}

Marinello F., Proto A.R., Zimbalatti G., Pezzuolo A., Cavalli R., Grigolato

S., 2017. Determination of forest road surface roughness by Kinect depth imaging. Ann. For. Res. 60(2): 217-226.

Abstract. Roughness is a dynamic property of the gravel road surface that affects safety, ride comfort as well as vehicle tyre life and maintenance costs. A rapid survey of gravel road condition is fundamental for an effective maintenance planning and definition of the intervention priorities. Different non-contact techniques such as laser scanning, ultrasonic sensors and photogrammetry have recently been proposed to reconstruct three-dimensional topography of road surface and allow extraction of roughness metrics. The application of Microsoft Kinect ${ }^{\mathrm{TM}}$ depth camera is proposed and discussed here for collection of 3D data sets from gravel roads, to be implemented in order to allow quantification of surface roughness. The objectives are to: i) verify the applicability of the Kinect sensor for characterization of different forest roads, ii) identify the appropriateness and potential of different roughness parameters and iii) analyse the correlation with vibrations recoded by 3 -axis accelerometers installed on different vehicles. The test took advantage of the implementation of the Kinect depth camera for surface roughness determination of 4 different forest gravel roads and one well-maintained asphalt road as reference. Different vehicles (mountain bike, off-road motorcycle, ATV vehicle, 4WD car and compact crossover) were included in the experiment in order to verify the vibration intensity when travelling on different road surface conditions. Correlations between the extracted roughness parameters and vibration levels of the tested vehicles were then verified. Coefficients of determination of between 0.76 and 0.97 were detected between average surface roughness and standard deviation of relative accelerations, with higher values in the case of lighter vehicles. Keywords low traffic volume road, surface roughness, vibrations, RGBdepth camera

Authors. Francesco Marinello - Department of Land, Environment, Agriculture and Forestry, University of Padova, Legnaro, Italy; Andrea Rosario Proto, Giuseppe Zimbalatti - Department of Agriculture, Mediterranean University of Reggio Calabria, Feo di Vito 89122, Reggio Calabria, Italy; Andrea Pezzuolo, Raffaele Cavalli, Stefano Grigolato (stefano.grigolato@unipd.it) - Department of Land, Environment, Agriculture and Forestry, University of Padova, Legnaro, Italy.

Manuscript received August 28, 2017; revised November 11, 2017; accepted November 16, 2017; online first November 16, 2017. 


\section{Introduction}

Forest roads are a fundamental requisite to connect woodlands with primary roads (Laschi et al. 2016). As a consequence, the role of a forest road network in providing access for harvesting operations is well recognized, but also for wildlife and fish habitats management, fire control, search and rescue operations and recreational activities (Akay \& Sessions 2004).

Traffic volumes, traffic speed and surfacing gravel properties and thicknesses are the main variables influencing the deterioration rates of gravelled forest roads (Grigolato et al. 2013, Huntington \& Ksaibati 2007) as well climate change and modification of the rain intensity (Aursand \& Horvli 2009, Kalantari \& Folkeson 2013).

Despite their importance, the maintenance of forest roads is often overlooked (Pellegrini et al. 2013). In particular, road surface maintenance is often neglected, with the consequent rapid degradation of the base course by formation of potholes, surface ruts or corrugations (Mills et al. 2007) and increment of the sediment production (Reid \& Dunne 1984, Thompson et al. 2010).

A good gravel road maintenance level is also fundamental in areas with a strong propensity towards nature-based tourism and recreational activities (Sitzia et al. 2016) as well for rapid search and rescue responses by ground transportation such as 4WD vehicles or All-Terrain Vehicles (ATV) (Ciesa et al. 2014, Lischke et al. 2001).

As a consequence, also due to the increasing tourism in forests, there is a need to periodically assess the surface quality of the forest road network.

Annual visual assessments are used to identify and prioritize periodic interventions to maintain the forest road network. However, these are costly and time consuming (Van der Gryp \& van Zyl 2007). For this reason, in the last years, several technologies and methods have been explored and proposed for a rapid survey of the surface condition of gravel roads. These approaches have mainly been based on distance measurement, vibration measurements, thermal cameras and ground penetrating radar (Romano et al. 2017)

A variety of instruments are currently available for the imaging of relatively large areas (Dugarsuren \& Lin 2016, Romans et al. 2017, Torresan et al. 2016, Talbot et al. 2017); with specific reference to stretches of road surfaces, two main approaches are widely recognised: two-dimensional and three-dimensional characterizations.

Two-dimensional imaging (Chambon \& Moliard 2011) takes advantage of low-cost CCD or CMOS based cameras in order to collect RGB or multispectral images (Dubbini et al. 2017) allowing dense acquisitions (down to the millimetre) with relatively large fields of view (up to some square metres) and with high scan rates (tens of frames per second) that allows high scanning speeds (up to $20-30 \mathrm{~m} / \mathrm{s}$ ). However, images are often not sufficiently well contrasted, with a poor information content, and robust algorithms are needed to detect, segment and quantify surface features.

Three-dimensional imaging (Mathavan 2015) encompasses different technologies, mainly ascribable to laser triangulation (Cigada et al. 2010), laser time of flight (Sanda \& Jucan 2013) and digital photogrammetry (Tiong et al. 2012). Such systems increase the information content: indeed, the reconstruction of local surface texture elevation is useful to better localize, isolate and measure surface features such as potholes, cracks, patches, etc. However, 3D instruments are more expensive, produce heavy point clouds that limit scan rates and are sensitive to noise and vibrations that may reduce the $3 \mathrm{D}$ reconstruction quality. In this paper implementation of the new Microsoft Kinect ${ }^{\mathrm{TM}}$ RGB-depth sensor is introduced and discussed. Due to its high resolution, fast scan rate and low cost (less than $100 €)$, this technology is gaining attention in many fields, such as agriculture (Marinello et al. 2015), speleology (Hämmerle et al. 2014) 
or automotive (Sadale et al. 2013).

The objective of the work is to verify the: (i) performances of the 3D Kinect depth image sensor to support quantification of surface roughness of different gravel roads, (ii) appropriateness and potential of different derivable roughness parameters and (iii) correlation between the derived roughness parameters and the vibrations recorded by 3 -axis accelerometers installed on different vehicles.

\section{Materials and methods}

\section{Experiment description}

The experimental sites were located in the Leogra Valley in north-eastern Italy. Specifically, one well-maintained asphalt road, four unpaved roads were identified and selected according to the following conditions: (i) homogeneity in terms of road surface roughness and slope for at least $100 \mathrm{~m}$, (ii) straightness or presence of curves with radii higher than 120 $\mathrm{m}$, (iii) local slope lower than $3 \%$ and average road slope lower than 5\%, (iv) accessibility to 4 wheeled vehicles.

The asphalt road was selected as reference, while the unpaved roads were: three unpaved roads with an surface improved by gravel and aggregate (gravel roads) and one unpaved road formed by compacted natural soil (dirt road). In order to have a high representation of gravel surfaces, the gravel roads were identified on the basis of different gravel size (prevalent gravel size $<15 \mathrm{~mm}$ - fine gravel; prevalent gravel size between 15-45 mm - medium gravel; prevalent gravel size $>45 \mathrm{~mm}$ - large gravel).

\section{Depth sensing camera technology}

Road surfaces were analysed using a Kinect depth camera: this instrument is based on the implementation of an infrared (IR) projector that shines an IR speckle pattern onto the road surface. Light back-reflected from the road interface is revealed by an IR sensor and comparison with the initial pattern is used to create a disparity map that then allow three-dimensional reconstruction of the surface.

Due to its technology and pixel resolution, the main performances of the Kinect sensor are influenced by the distance of the instrument from the target surface, as shown in Table 1. During tests the instrument was positioned at an average height of $1 \mathrm{~m}$ above the mean road plane, this being the ideal trade-off between measuring range and resolution (sufficiently high to allow characterization of different road features).

All of the roads were analysed in ten different transects spaced $10 \mathrm{~m}$ apart. In each transect, three different positions were identified (one in the centre and two on the sides) and scanned by the Kinect sensor, for a total of 30 measurements.

\section{Calibration and parameters}

The Kinect sensor was implemented to allow 3D characterization of road interfaces. Collected depth scans were filtered in order to remove installation slope from 3D data sets and then processed in order to estimate quantitative roughness parameters. Specifically, over

Table 1 Sensor scanning performance

\begin{tabular}{llll}
\hline $\begin{array}{l}\text { Distance } \\
(\mathrm{mm})\end{array}$ & $\begin{array}{l}\text { Lateral range } \\
(\mathrm{mm})\end{array}$ & $\begin{array}{l}\text { Lateral resolution } \\
(\mathrm{mm})\end{array}$ & $\begin{array}{l}\text { Vertical resolution } \\
(\mathrm{mm})\end{array}$ \\
\hline 800 & $625 \times 470$ & $0.95 \times 0.95$ & 1.6 \\
\hline 1000 & $730 \times 550$ & $1.15 \times 1.15$ & 2.0 \\
\hline 1200 & $833 \times 630$ & $1.30 \times 1.30$ & 2.4 \\
\hline 1400 & $935 \times 710$ & $1.45 \times 1.45$ & 2.9 \\
\hline
\end{tabular}


30 different parameters were selected and tested, as defined by international standard ISO 25178-2 (2012). The aim of the study was to identify those parameters that have a higher discriminative potential. To this end, for each parameter $S x$ the average variability $V_{S x}$ was computed as follows:

$V_{S x}=\frac{\sum_{i=1}^{5} \sigma_{i}(S x)}{5 \cdot \Delta(S x)}$

where $\sigma$ indicates the standard deviations of the 5 analyzed roads, and $\Delta$ indicates the maximum difference between the $S x$ values of the same roads. Parameters $S x$ exhibiting the lowest $V_{S x}$ values were selected and analyzed, also in comparison with vehicle vibrations. The following parameters were then considered: $\mathrm{Sa}$, Sq, Spk, Sk and Svk.

Assuming that Kinect provides a $x-y-z$ cloud of points, each representing the local vertical elevation $z$ in a given road position identified by $x, y$ horizontal coordinates, $\mathrm{Sa}$ is the arithmetical mean of absolute deviations of the ordinate values from the mean plane, within a definition area $A$, and is defined by (2):

$S a=\frac{1}{A} \iint_{A}|z(x, y)| \mathrm{dxdy}$

calculated after mean plane subtraction.

Similarly, $S q$ is the root mean square value of the deviations of the ordinate values from the mean plane, and is defined by (3):

$S q=\sqrt{\frac{1}{A} \iint_{A} z^{2}(x, y) d x d y}$

$S p k, S k$ and $S v k$ are three-dimensional stratified parameters, estimated on the basis of the bearing area ratio curve. $S k$ is a parameter associated to the core roughness of the 3D surface, where the load is mainly distributed by passing vehicles. Spk is the reduced peak height, representing the height distribution over the core roughness; $S v k$ is the reduced valley depth quantifying depth variations under the core roughness.

Roughness parameters were estimated after 220 filtering, performed through removal of the second degree best fitting polynomial curve: this post processing operation had the scope of minimizing the influence of instrument installation slope and road curvatures on final analyses.

In order to evaluate the discriminative potential of roughness parameters, the collected parameter values estimated in the 30 different positions for each road were statistically compared through an analysis of variance (ANOVA) by the test $F$ at a significance level of $p$ $<0.01$, while the means were compared by a post hoc Tukey's test at 5\% probability of error.

\section{Vehicles and vibrations}

Five different vehicles were chosen to cover the range of models typically driven on forest roads. To this end a mountain bike, an off-road motorcycle, an all-terrain vehicle, a four-wheel drive car and a compact crossover were included in the experiment: their speed was set to 18 $\mathrm{km} \mathrm{h}^{-1}\left(5 \mathrm{~m} \mathrm{~s}^{-1}\right)$, which is considered to be an acceptable speed for common transportation means on different forest road conditions.

The main characteristics of the vehicles are reported in the table below. In order to monitor and study the effects of different road surfaces on vehicle stability, the intensity of vibrations was monitored by means of two accelerometers MSR 165 (MSR Electronics Gmbh), positioned as described in the following Table 2. The devices contain high resolution 3 -axis acceleration sensors with a working range as high as $\pm 200 \mathrm{~g}$; they are small and light enough ( $\sim 69$ grams) to be mounted on the vehicles without interfering with their dynamics. The logger was configured to start acquiring data at a $100 \mathrm{~Hz}$ sampling rate, during a $20 \mathrm{~s}$ travel duration. Each experiment-travel with each vehicle for each road was repeated ten times on the same road sections with the same driver, in order to exclude or minimize variability arising from different driver weights and postures. 
Table 2 Main characteristics of the vehicles

\begin{tabular}{lllll}
\hline Vehicle & Model & $\begin{array}{l}\text { Weight including } \\
\text { driver }(\mathrm{kg})\end{array}$ & $\begin{array}{l}\text { Power } \\
(\mathrm{kW})\end{array}$ & $\begin{array}{l}\text { Vibration sensor } \\
\text { positions }\end{array}$ \\
\hline Mountain bike & GT, Avalanche & 90 & - & $\begin{array}{l}\text { 1) handlebar } \\
\text { 2) saddle }\end{array}$ \\
\hline Off-road motorcycle & KTM, Sx & 170 & 24 & $\begin{array}{l}\text { 1) handlebar } \\
\text { 2) saddle }\end{array}$ \\
\hline ATV & Polaris, Sportsman & 450 & 38 & $\begin{array}{l}\text { 1) handlebar } \\
\text { 2) saddle }\end{array}$ \\
\hline 4WD car & FIAT, Panda 4x4 & 1040 & 40 & $\begin{array}{l}\text { 1) front axle } \\
\text { 2) rear seat }\end{array}$ \\
\hline Compact crossover & Subaru, Forester & 1620 & 92 & $\begin{array}{l}\text { 1) front axle } \\
\text { 2) rear seat }\end{array}$ \\
\hline
\end{tabular}

Once data acquisition was completed, the logger was attached to a PC via USB interface to allow data extraction for further analysis. Vibrations were quantified as the root mean square of the oscillations in terms of relative accelerations, as reported in equation (4):

$R M S=\frac{1}{g} \sqrt{\sum_{i=1}^{N}\left(a_{x, i}^{2}+a_{y, i}^{2}+\left(a_{z, i}-g\right)^{2}\right)}$

where $N$ is about 2000 and represents the total number of points collected in the $20 \mathrm{~s}$ travel duration, $g=9.81 \mathrm{~m} \mathrm{~s}^{-2}$ is the standard gravity acceleration and $a_{x, i}, a_{y, i}$ and $a_{z, i}$ are the accelerations measured in the three coordinate axes ( $x$ parallel, $y$ transversal and $z$ perpendicular with respect to road direction).

Root mean square (RMS) was then correlated to different roughness parameters through a linear regression. Coefficient of determination $\mathrm{R}^{2}$ and slope $\mathrm{m}$ were concurrently quantified: the first as an indicator of the predictive power of the model, and the second to estimate the sensitivity of RMS to roughness parameters variations.

\section{Results}

\section{Roughness analysis}

Before starting roughness analysis, the Kinect sensor underwent a preliminary calibration op- eration, performed on reference hemispherical surfaces placed on a flat surface (see Figure 1a and $1 b$ ) with an average roughness $S a<0.20$ $\mathrm{mm}$, as described in (Marinello et al. 2015). The instrument was then applied to characterize 5 roads as discussed in previous sections, as for instance shown in Figure 1c-11. Measurements were taken immediately before vehicle transits. On the fine gravel road test measurements were also repeated after vehicle transits and it was verified that the traffic in the proposed tests was sufficiently low not to modify road average roughness.

All roads underwent roughness analyses: the main results are reported in Table 3. It can be noted that in all cases the asphalt road exhibits the lowest roughness values and, conversely, the large gravel roads the highest ones. Stones present in the large gravel road cause an average increase of vertical elevation variations, which can be estimated as two-three times higher than on the asphalt road. The dirt road exhibits values for the different parameters that are in general comparable to the asphalt and fine gravel roads. Indeed, the roughness parameters are not influenced by interface material but rather quantify gravel or stone sizes as well as unpaved terrain unevenness.

The overall mean values of analysed roughness parameters (namely $S a, S q, S p k, S k$ and $S v k)$ were in all cases significantly different (ANOVA test: $F>50, F_{\text {crit }}=2.43, p<0.01$ ). 

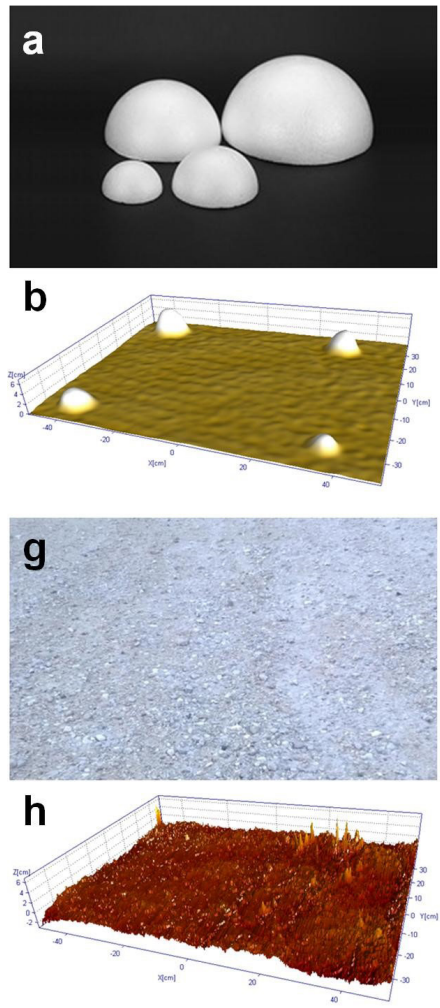
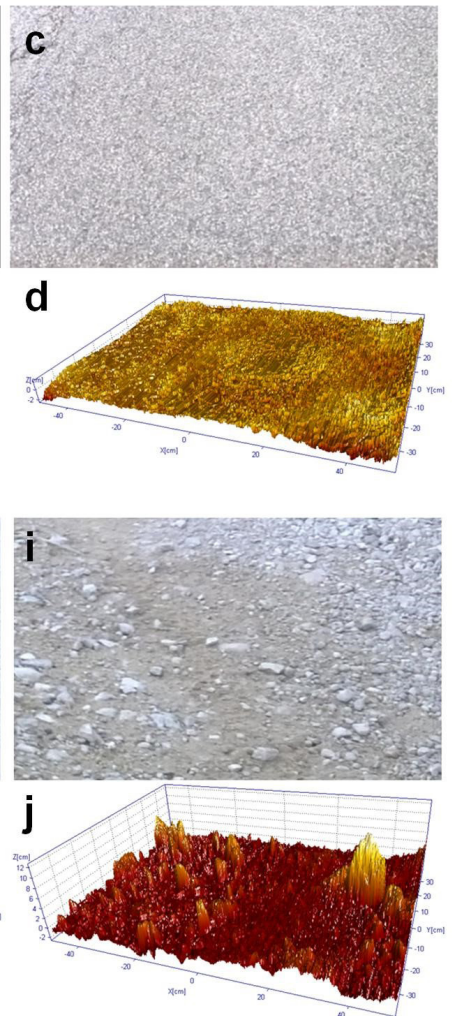
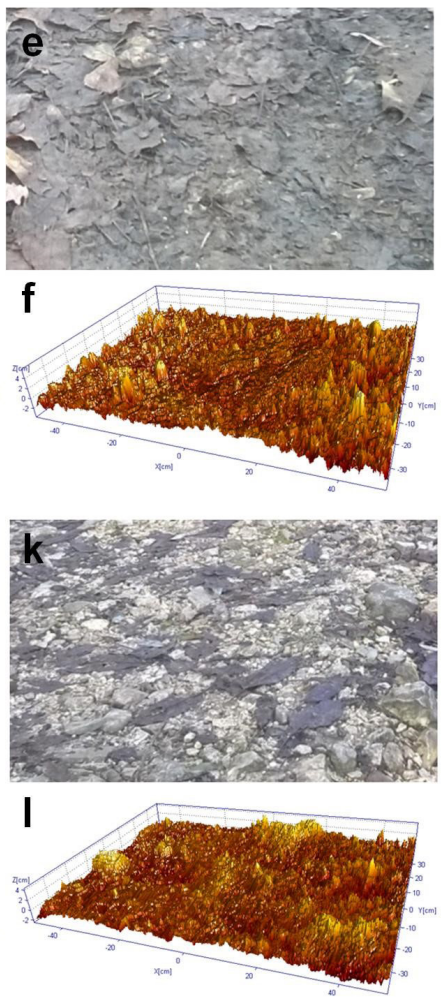

Figure 1 Three-dimensional Kinect reconstruction of different surfaces: a-b) reference hemispheres used for instrument calibration; c-d) asphalt road; e-f) dirt road; g-h) fine gravel road; i-j) medium gravel road and k-1) large gravel road.

Furthermore, all the parameters highlighted discriminative potential for the asphalt road and the three fine, medium and large gravel roads. Only the dirt road had a variable behaviour in terms of surface roughness parametrization, as highlighted by the Tukey test results and expressed by the same letters in Table 3.

\section{Vibrations analysis}

As stated above, this study also aimed at analysing the correlation between roughness analyses and vibrations recoded by 3 -axis accelerometers installed on different vehicles. The main results are reported in the graphs below (Figure 2). For the sake of simplicity graphical results are reported only with reference to the 222 mean roughness $S a$, while for the other roughness parameters relevant values are given in Table 4.

It can be noted that there is a clear correlation between road roughness and vehicle vibrations: the higher the roughness due to gravel or stones is, the larger is the amount of vibration transmitted from the wheels to the different parts of the vehicle. The slopes of the functions achieved after linear regression characterize the entity of this relationship: the steeper the slope is, the higher is the sensitivity of the vehicle to the presence of irregularities on unpaved roads. For example, compared to an off-road motorcycle or an all-terrain vehicle, a mountain bike handlebar is four times more sensitive to vibrations: this can be ascribed to the weight of the vehicle, number and size of 
Table 3 Main roughness parameters and statistical analyses results for the five analysed roads. Values in brackets indicate standard deviations on 30 samples. Same letters do not have statistically significant difference with a $5 \%$ probability in a Tukey test.

\begin{tabular}{llllll}
\hline Road state & $S a(\mathrm{~mm})$ & $S q(\mathrm{~mm})$ & $S p k(\mathrm{~mm})$ & $S k(\mathrm{~mm})$ & $S v k(\mathrm{~mm})$ \\
\hline Asphalt & $1.28(0.10)^{\mathrm{a}}$ & $1.63(0.14)^{\mathrm{a}}$ & $2.12(0.29)^{\mathrm{a}}$ & $4.07(0.29)^{\mathrm{a}}$ & $1.73(0.22)^{\mathrm{a}}$ \\
\hline Dirt & $1.66(0.26)^{\mathrm{b}}$ & $2.30(0.40)^{\mathrm{b}}$ & $4.19(0.84)^{\mathrm{b}}$ & $4.52(0.57)^{\mathrm{b}}$ & $2.42(0.58)^{\mathrm{b}}$ \\
\hline Fine gravel & $1.65(0.17)^{\mathrm{b}}$ & $2.15(0.24)^{\mathrm{b}}$ & $3.30(0.45)^{\mathrm{c}}$ & $5.05(0.50)^{\mathrm{c}}$ & $2.08(0.23)^{\mathrm{c}}$ \\
\hline Medium gravel & $2.03(0.24)^{\mathrm{c}}$ & $2.76(0.37)^{\mathrm{c}}$ & $4.94(0.79)^{\mathrm{d}}$ & $5.84(0.52)^{\mathrm{d}}$ & $2.62(0.39)^{\mathrm{b}}$ \\
\hline Large gravel & $2.79(0.55)^{\mathrm{d}}$ & $3.75(0.77)^{\mathrm{d}}$ & $6.14(1.47)^{\mathrm{e}}$ & $8.15(1.52)^{\mathrm{e}}$ & $3.83(0.82)^{\mathrm{d}}$ \\
\hline Test $F$ value & 73.1 & 68.7 & 56.4 & 77.1 & 68.7 \\
\hline
\end{tabular}

the wheels (radius and width), or shock absorbers effectiveness. Similarly, the sensitivity to soil roughness of the vibrations at the (rear) seat of the vehicles exhibits a decrease with the increase in weight: i.e. heavier vehicles are less sensitive to variations in road irregularities. Such dependencies are supported by high correlations in general with $R^{2}>0.7$. Slightly lower values were achieved only in the case of the off-road motorcycle: this is most probably due to the tyre profile which was relatively large and could have introduced additional vibrations during travelling. The best results were in the case of the $S q$ roughness parameter, with a coefficient of determination as high as $R^{2}=0.91$ on average and as high as $R^{2}=$ 0.98 in the case of the ATV handlebar and $R^{2}=$ 0.97 in the case of the 4WD car rear seat.

\section{Discussion}

At present there is a growing need for fast and accurate methods allowing quantitative characterization of road surfaces. The approach proposed in the present paper, which combine Kinect 3D and vibrations analyses, can be a cost effective solution. In particular, specific roughness parameters such as $S p k$ and $S k$ can properly discriminate different roads conditions, providing a robust reference for monitoring and subsequent classification.

The potential advantage of the proposed method is the possibility to create a decision support model able to predict the maintenance requirements of low traffic roads and forest road networks according to different vehicles and the prevalent use of the network.

Indeed, regression analyses between different roughness parameters and vibration intensities allowed definition of models which can be used in two different ways. Firstly, the roughness of a road surface can be estimated on the basis of vibration intensities recorded by vehicles during travel. Therefore, vehicles equipped with proper accelerometers and driven on forest roads can be used as on the go platforms for the characterization of the condition of stretches of road surfaces, road ride comfort as well road durability (Nitsche et al. 2014). This is useful for identification of critical areas and for providing effective decision support for short and medium maintenance scheduling (Forslöf \& Jones 2015). Secondly the knowledge of the roughness of specific roads can be implemented to help prediction of vibration intensities on different vehicles. Since induced vibrations are connected to several safety issues for the drivers and for the vehicles themselves, the availability of roughness data can allow an a priori definition of driving conditions (for instance in terms of maximum speed) and of access time (for instance in term of average speed and the consequent average time to travel one distance unit as suggested by Ciesa et al. 2014).

In this work, it should be noted how acquired data are evidently affected by high variability: this is something which is very typical of vibration studies as well as roughness analyses 

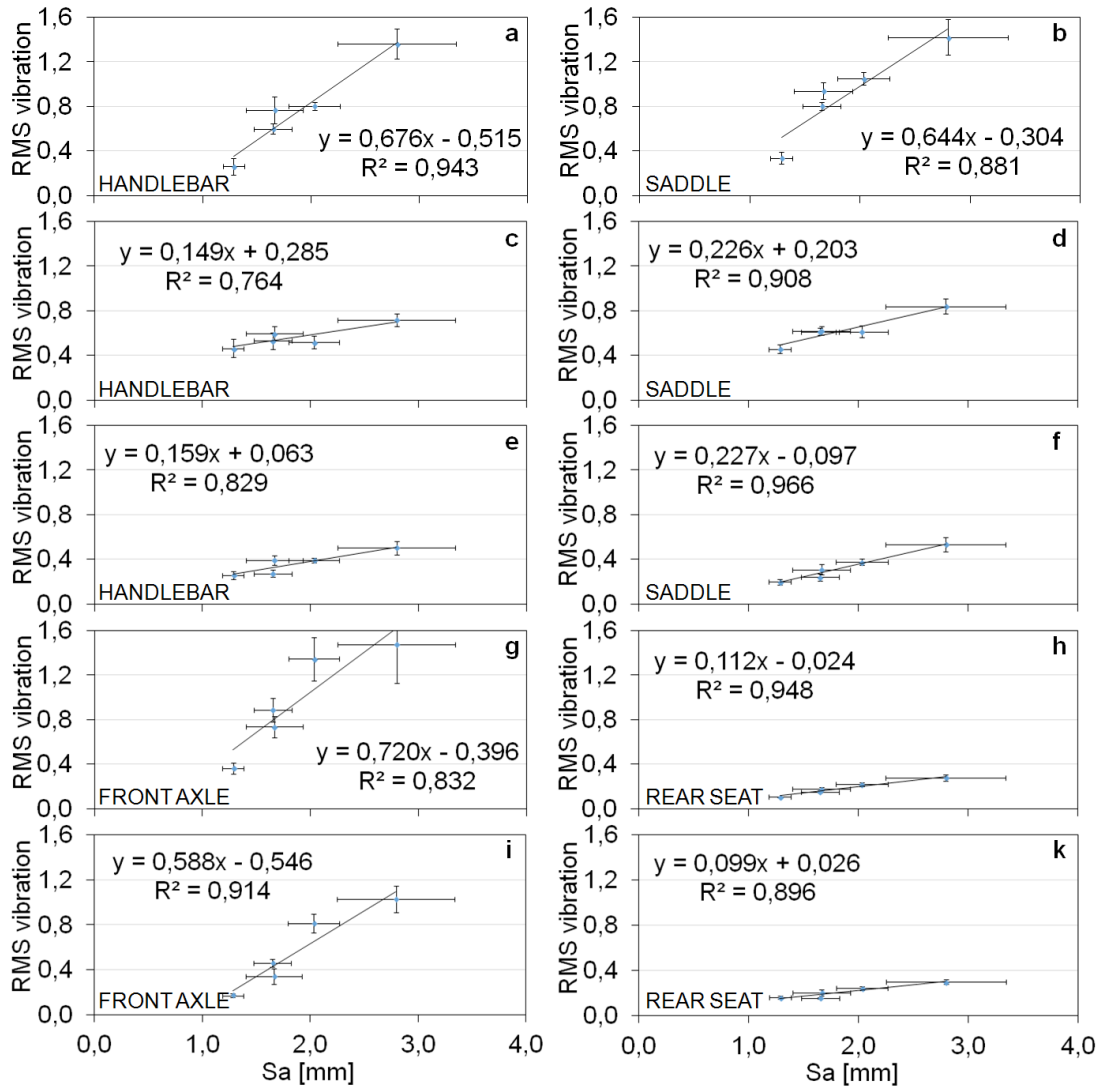

Figure 2 Root mean square relative vibrations reported as a function of average roughness Sa for different positions in different vehicles: $a-b)$ mountain bike, c-d) off-road motorcycle, e-f) all-terrain vehicle, g-h) four-wheel drive car and $\mathrm{i}-\mathrm{j}$ ) compact crossover.

Table 4 Correlation analyses between different positions of different vehicles RMS vibrations and soil roughness parameters; in each column $m$ indicates the slope and $\mathrm{R}^{2}$ the coefficient of determination after linear regression.

\begin{tabular}{|c|c|c|c|c|c|c|}
\hline Vehicle & Position & $\begin{array}{l}S a \\
m-R^{2}\end{array}$ & $\begin{array}{l}S q \\
m-R^{2}\end{array}$ & $\begin{array}{l}S p k \\
m-R^{2}\end{array}$ & $\begin{array}{l}\text { Ssk } \\
m-R^{2}\end{array}$ & $\begin{array}{l}S v k \\
m-R^{2}\end{array}$ \\
\hline \multirow{2}{*}{$\begin{array}{l}\text { Mountain } \\
\text { bike }\end{array}$} & handlebar & $0.644-0.881$ & $0.472-0.916$ & $0.252-0.969$ & $0.216-0.779$ & $0.461-0.880$ \\
\hline & saddle & $0.676-0.943$ & $0.491-0.963$ & $0.252-0.941$ & $0.230-0.862$ & $0.491-0.970$ \\
\hline \multirow{2}{*}{$\begin{array}{l}\text { Off-road } \\
\text { motorcycle }\end{array}$} & handlebar & $0.226-0.908$ & $0.163-0.911$ & $0.081-0.843$ & $0.078-0.859$ & $0.163-0.917$ \\
\hline & saddle & $0.149-0.764$ & $0.108-0.774$ & $0.054-0.708$ & $0.051-0.696$ & $0.113-0.858$ \\
\hline \multirow{2}{*}{ ATV } & handlebar & $0.227-0.966$ & $0.165-0.982$ & $0.084-0.943$ & $0.078-0.900$ & $0.164-0.981$ \\
\hline & saddle & $0.159-0.829$ & $0.117-0.874$ & $0.063-0.925$ & $0.052-0.707$ & $0.119-0.910$ \\
\hline \multirow{2}{*}{ 4WD car } & front axle & $0.720-0.832$ & $0.522-0.845$ & $0.274-0.865$ & $0.250-0.790$ & $0.483-0.729$ \\
\hline & rear seat & $0.112-0.948$ & $0.081-0.974$ & $0.043-0.989$ & $0.038-0.866$ & $0.079-0.932$ \\
\hline \multirow{2}{*}{$\begin{array}{l}\text { Compact } \\
\text { crossover }\end{array}$} & front axle & $0.588-0.914$ & $0.422-0.911$ & $0.213-0.862$ & $0.208-0.900$ & $0.398-0.816$ \\
\hline & rear seat & $0.099-0.896$ & $0.072-0.919$ & $0.037-0.901$ & $0.034-0.823$ & $0.072-0.923$ \\
\hline
\end{tabular}


(Abulizi et al. 2016). However, the authors believe this does not limit the proposed analysis approach in a substantial way. Indeed, on the one hand accelerometers can collect hundreds of acceleration values per second, allowing a big data set to overcome limitations connected with the intrinsic variability of the measures. On the other, the Kinect sensor proposed for $3 \mathrm{D}$ reconstruction can also provide analyses with a frame rate as high as $30 \mathrm{fps}$, thus again allowing collection of big data sets to minimize the influence of road roughness variability.

The fully exploitation of the method is connected with the definition of regression models with other different vehicles, and possibly with different travelling speeds. As a consequence, further standardised tests on vehicle vibration and forest road surface roughness analyses are still indispensable to strengthen the proposed method and to extend it also to forest machinery and different timber transport truck combinations.

\section{Conclusions}

This paper concerns application of the Microsoft Kinect ${ }^{\mathrm{TM}}$ to reconstruct three-dimensional topography of forest road surface and extract roughness metrics. The roughness parameters have then been compared to the vibration intensity recorded by 3-axis accelerometers installed on different vehicles.

The analysis highlighted a clear relationship between different calculated roughness parameters and the vibration intensities for the different tested vehicles. Specifically, in the cases of roughness parameters $S a$ and $S q$, coefficients of determination in general higher than 0.75 were detected for all road conditions, supporting the applicability of the proposed approach for analyses on road conditions and relative effects on travelling vehicles.

\section{Acknowledgements}

The authors wish to thank Mr. Piero Dalle Molle and Mr. Manfredi De Bernardo for the field activities and data acquisitions.

\section{References}

Abulizi N., Kawamura A., Tomiyama K., Fujita, S. 2016. Measuring and evaluating of road roughness conditions with a compact road profiler and ArcGIS. Journal of Traffic and Transportation Engineering 3(5): 398-411. DOI: $10.1016 /$ j.jtte.2016.09.004

Akay A.E., Sessions J., 2004. Roading and transport operations. Encyclopedia of Forest Sciences (193): 259-269.

Aursand P.O., Horvli I., 2009. Effect of a changed climate on gravel roads. In 8 th International Conference on the Bearing Capacity of Roads, Railways and Airfields BCR2A 2009: 1091-1100.

Chambon S., Moliard J.M., 2011. Automatic road pavement assessment with image processing: Review and comparison, International Journal of Geophysics 2011: 1-20. DOI: $10.1155 / 2011 / 989354$

Ciesa M., Grigolato S., Cavalli, R., 2014. Analysis on vehicle and walking speeds of search and rescue ground crews in mountainous areas. Journal of Outdoor Recreation and Tourism 5-6: 48-57. DOI:10.1016/j. jort.2014.03.004

Cigada A., Mancosu F., Manzoni S., Zappa E., 2010. Laser-triangulation device for in-line measurement of road texture at medium and high speed, Mechanical Systems and Signal Processing 24(7): 2225-2234. DOI: 10.1016/j.ymssp.2010.05.002

Dubbini M., Pezzuolo A., De Giglio M., Gattelli M., Curzio L., Covi D., Yezekyan T., Marinello F., 2017. Last generation instrument for agriculture multispectral data collection. CIGR Journal 19(1): 87-93.

Forslöf L., Jones H., 2015. Roadroid: Continuous road condition monitoring with smart phones. Journal of Civil Engineering and Architecture 9(4): 485-496. DOI:10.17265/1934-7359/2015.04.012

Grigolato S., Pellegrini M., Cavalli R., 2013. Temporal analysis of the traffic loads on forest road networks. iForest - Biogeosciences and Forestry 6(4): 255-261. DOI:10.3832/ifor0773-006

Hämmerle M., Höfle B., Fuchs J., Schröder-Ritzrau A., Voll-weiler N., Frank N., 2014. Comparison of Kinect and terrestrial Lidar capturing natural karst cave 3-D objects, IEEE Geoscience and Remote Sensing Letters 11(11): 1896-1900. DOI: 10.1109/LGRS.2014.2313599

Huntington G., Ksaibati K., 2007. Gravel roads surface performance modelling. Transportation Research Record 2016: 56-64. DOI:10.3141/2016-07

ISO 25178-2: 2012 Geometrical Product Specifications (GPS) - Surface texture: Areal - Part 2: Terms, defini- 
tions and surface texture parameters. International Standards Organization, Switzerland.

Kalantari Z., Folkeson L. 2013. Road drainage in Sweden: Current practice and suggestions for adaptation to climate change. Journal of Infrastructure Systems 19(2): 147-156. DOI:10.1061/(ASCE)IS.1943-555X.0000119

Laschi A., Neri F., Montorselli N.B., Marchi, E., 2016. A methodological approach exploiting modern techniques for forest road network planning. Croatian Journal of Forest Engineering 37(2): 319-331.

Lischke V., Byhahn C., Westphal K., Kessler P. 2001. Mountaineering accidents in the European Alps: Have the numbers increased in recent years? Wilderness and Environmental Medicine 12(2): 74-80.

Marinello F., Pezzuolo A., Gasparini F., Arvidsson J., Sartori L., 2015. Application of the Kinect sensor for dynamic soil surface characterization. Precision Agriculture 16(6): 601-612. DOI: 10.1007/s11119-015-9398-5

Mathavan S., Kamal K., Rahman M., 2015. A review of three-dimensional imaging technologies for pavement distress detection and measurements, IEEE Transactions On Intelligent Transportation Systems 16(5): 2353-2362. DOI: 10.1109/TITS.2015.2428655

Mills K., Pyles M., Thoreson R. 2007. Aggregate surfacing design and management for low-volume roads in temperate, mountainous areas. Transportation Research Record. Journal of the Transportation Research Board 1989: 154-160. DOI:10.3141/1989-59

Nitsche P., Stütz R., Kammer M., Maurer P. 2014. Comparison of Machine Learning Methods for Evaluating Pavement Roughness Based on Vehicle Response. Journal of Computing in Civil Engineering 28(4): 04014015. DOI:10.1061/(ASCE)CP.1943-5487.0000285

Sanda N.S.R. 2013. Aspects regarding 3D laser scanning surveys for road design. Agricultura 85(1-2): 140-144. DOI: 10.15835/arspa.v85i1-2.10014

Pellegrini M., Grigolato S., Cavalli R., 2013. Spatial multi-criteria decision process to define maintenance priorities of forest road network: an application in the Italian Alpine region. Croatian Journal of Forest Engineering 34(1): 31-42.

Reid L.M., Dunne T. 1984. Sediment production from forest road surfaces. Water Resources Research 20(11): 1753-1761.

Roman A., Ursu T.-M., Fărcaş S., Lăzărescu V.-A., Opreanu C.H., 2017. An integrated airborne laser scanning approach to forest management and cultural heritage issues: A case study at Porolissum, Romania. Annals of Forest Research 60(1): 171-187. DOI: 10.15287/ afr.2016.755

Romano A., Costa A., Basile M., Raimondi R., Posillico M., Scinti Roger D., Crisci A., Piraccini R., Raia P., Matteucci G., 2017. Conservation of salamanders in managed forests: Methods and costs of monitoring abundance and habitat selection. Forest Ecology and Management 400: 12-18. DOI:10.1016/j.foreco.2017.05.048

Sadale R., Kolhe R., Wathore S., Aghav J., Warade S., Udayagiri S., 2013. Steer-by-wire implementation using Kinect, Advances in Intelligent Systems and Computing. In: Kumar M. A., R. S., Kumar T. (eds) Proceedings of International Conference on Advances in Computing. Advances in Intelligent Systems and Computing, vol 174. pp 163-170. Springer, New Delhi. DOI: 10.1007/978-81-322-0740-5_21

Sitzia T., Campagnaro T., Grigolato S. 2016. Ecological risk and accessibility analysis to assess the impact of roads under Habitats Directive. Journal of Environmental Planning and Management 59(12): 2251-2271. DOI: 10.1080/09640568.2016.1140023

Talbot B., Pierzchała M., Astrup, R. 2017. Applications of remote and proximal sensing for improved precision in forest operations. Croatian Journal of Forest Engineering 38(2): 327-336.

Thompson M., Sessions J., Boston K., Skaugset A., Tomberlin D. 2010. Forest road erosion control using multiobjective optimization. JAWRA Journal of the American Water Resources Association 46(4): 712-723. DOI:10.1111/j.1752-1688.2010.00443.x

Tiong L.T.Y., Mustaffar M., Hainin M.R., 2012. Road surface assessment of pothole severity by close range digital photogrammetry method. World Applied Sciences Journal 19(6): 867-873. DOI: 0.5829/idosi. wasj.2012.19.06.3353

Torresan C., Corona P., Scrinzi G., Marsal J.V., 2016. Using classification trees to predict forest structure types from LiDAR data. Annals of Forest Research 59(2): 281-298. DOI: 10.15287/afr.2016.423

Van der Gryp A., Van Zyl G., 2007. Variability and control of gravel road visual assessments. Transportation Research Record: Journal of the Transportation Research Board, 1989(1989): 247-253. DOI:10.3141/1989-70 